\title{
Evaluation of Acceleration-Based Disturbance Observation for Multicopter Control
}

\author{
Teodor Tomić
}

\begin{abstract}
Small UAVs flying in narrow passages require robustness to turbulence caused by self-induced vortices. Aerial manipulation introduces modeling errors due to payload and parameter changes. When large external forces are applied, small helicopters must fly at orientations far outside hover conditions. The compensation of such uncertainties can be achieved through disturbance observation (DO). An onboard IMU makes the platform well-suited for acceleration-based DO. In this paper, we evaluate a cascaded attitude and position tracking controller for a quadrotor. Quaternions are used for attitude control to allow large orientation angles. We investigate attitude tracking by the boundary-layer integral sliding mode control coupled with acceleration-based DO. The position controller generates singularity-free quaternion and angular velocity signals. The presented controller is experimentally verified and compared to PID and backstepping controllers for trajectory tracking and hovering in turbulent conditions. Compensation of large external forces in the horizontal plane is shown through a stable $45^{\circ}$ hover.
\end{abstract}

\section{INTRODUCTION}

\section{A. Motivation}

Autonomous Unmanned Aerial Vehicles (UAVs) have seen a large rise in the number of applications in recent years. The fully autonomous execution of inspection and aerial manipulation tasks requires UAVs to operate in a wide variety of unknown environmental conditions, including wind gusts and vortices, and under uncertain or changing system parameters. Unknown environment forces can arise when a UAV is in contact with a static environment. If large external forces are present, large attitude angles are required for their compensation.

To compensate general uncertainties, disturbance observation (DO) can be utilized. Acceleration-based disturbance observation is well-suited for small UAVs because acceleration measurements are provided by the Inertial Measurement Unit (IMU). Attitude control typically runs at high update rate ( $1 \mathrm{kHz}$ in our case), so angular acceleration can be computed from the angular velocity by finite differences. A benefit of a DO over robust control is that it can directly estimate external forces from the system model. The estimate can also be used for environment interaction if no applicable sensors are available.

In our approach, we adopt a well-established method for disturbance observation [1], [2]. We use the system model to estimate the disturbance from the acceleration signal. In this way, stable response is obtained even in environment interaction applications. We investigate the boundary-layer integral

German Aerospace Center (DLR) Robotics and Mechatronics Center (RMC), Münchner Straße 20, 82234 Weßling, Germany. Corresponding author: teodor.tomicedlr.de
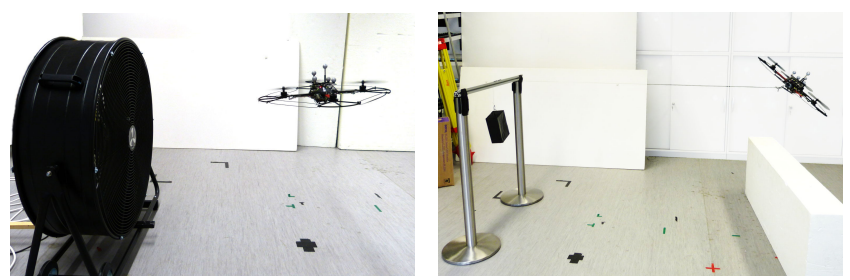

(a) Hovering in a $3 \mathrm{~m} / \mathrm{s}$ turbulent (b) Hovering with a constant side wind stream, generated by a fan force produced by a suspended with $70 \mathrm{~cm}$ diameter $6 \mathrm{~N}$ weight

Fig. 1: Experimental setups for hovering tests with external disturbance investigated in this paper.

sliding mode controller [3] with disturbance observation in the attitude tracking context. The boundary layer solution is a well-established method to eliminate chattering, where the controller behaves locally like a linear controller.

For our quadrotor UAV, we adopt a cascaded controller structure. The inner quaternion-based attitude controller is based on established methods [4], [5] and is suitable for large orientation angles. The outer Cartesian position controller converts a desired control force to a quaternion and angular velocity reference in a singularity-free way. The angular velocity is generated directly from the feedback signal, hence we do not rely on a trajectory feedforward signal. We show that using a DO in the attitude controller improves position controller performance in turbulent wind conditions. The improvement is also shown when the DO is used in the position controller. Influence of external disturbances is investigated as depicted in Fig. 1.

\section{B. Related work}

Cascaded PID [6], LQR [7] and feedback linearization controllers [8] provide adequate trajectory following performance about hover conditions. Outside hover conditions, attitude control can be done using geometric representations of the attitude error, for example by using rotation matrices [9] or quaternions [4]. Some of the techniques used for dealing with uncertainties are backstepping [10], sliding mode [11] control, $H_{\infty}$ control [12], model predictive control [13], and adaptive control [14], [15].

Explicit estimation of the uncertainties has previously been shown to improve flight performance. For this, sliding mode disturbance observers [16], [17] have been used. However, higher-order sliding modes are computationally expensive for embedded systems due to noninteger powers. The disturbance estimate must be filtered to avoid chattering, thereby sacrifing robustness. Adaptive Integral Backstepping Control, 
which estimates and compensates model uncertainties, has also been applied to quadrotors [18], [19]. The controller has been shown to be effective for varying system inertia. Due to the large number of parameters it is not easy to tune. In [20] a two-dimensional wind disturbance is estimated and compensated. Extended state observers have been applied for helicopter disturbance observation in [21], [22], in the active disturbance rejection control (ADRC). Kalman-filter-based disturbance observation has been shown for quadrotors [15] for near-hover conditions.

Acceleration-based DO has been shown to improve tracking performance of various systems, such as a tilt-wing quadrotor [23] and underwater vehicles [24]. In [25], the authors show performance improvement when using a disturbance observer for quadrotor attitude control. Significant improvements have not been shown when applied to position control. Investigation of disturbance observers in quadrotors has also been done in [26], [27], [28]. However, here only near-hover conditions were investigated. Experiments are limited, only showing attitude control. Euler angles were used for attitude representation. In this paper, we apply the DO to a quaternion-based attitude controller and show significant performance improvements in position control, as well as compensation of large external forces.

In the paper, we first present the system model before developing the attitude and position tracking controllers. Presentation of the simulation and experimental results is followed by the conclusion.

\section{SySTEM MODEL}

\section{A. Kinematics}

In this paper, we consider three coordinate frames: the non-moving inertial frame $I$, the body-fixed frame $B$, and the desired frame $D$. The goal of the attitude tracking controller is to align frame $B$ with frame $D$. Unit quaternions are used for the singularity-free attitude representation. The quaternion $\underline{\boldsymbol{q}}=\left[\eta \boldsymbol{\epsilon}^{T}\right]^{T}$ consists of the scalar part $\eta$ and the vector part $\boldsymbol{\epsilon}$. To prevent notational ambiguity with vectors, we denote quaternions with an underbar. Unit quaternion attitude representation is related to the angle-axis representation through the half of the rotation angle:

$$
\eta=\cos \frac{\varphi}{2}, \quad \boldsymbol{\epsilon}=\boldsymbol{k} \sin \frac{\varphi}{2}
$$

where $k$ is the rotation axis, and $\varphi$ the rotation angle. The norm of a unit quaternion is always unity such that $\|\underline{q}\|=$ $\left(\underline{\boldsymbol{q}}^{T} \underline{\boldsymbol{q}}\right)^{1 / 2}=1$. The conjugate of a unit quaternion $\underline{\boldsymbol{q}}^{*}=$ $\left[\eta-\boldsymbol{\epsilon}^{T}\right]^{T}$ represents inverse rotation. The rotation matrix $\boldsymbol{R}(\boldsymbol{q})$ can be obtained by using the Euler-Rodriguez formula [5]. The kinematic differential equation of a unit quaternion is

$$
\underline{\dot{q}}=\frac{1}{2} \boldsymbol{U}(\underline{\boldsymbol{q}}) \boldsymbol{\omega}=\frac{1}{2}\left[\begin{array}{c}
-\boldsymbol{\epsilon}^{T} \\
\eta \boldsymbol{I}_{3 \times 3}+\mathcal{S}(\boldsymbol{\epsilon})
\end{array}\right] \boldsymbol{\omega}
$$

where the skew-symmetric matrix operator $\mathcal{S}(\cdot)=-\mathcal{S}^{T}(\cdot)$ is defined such that $\mathcal{S}(\boldsymbol{a}) \boldsymbol{b}=\boldsymbol{a} \times \boldsymbol{b}$ and $\boldsymbol{\omega}=[p q r]^{T}$ is the body angular velocity.

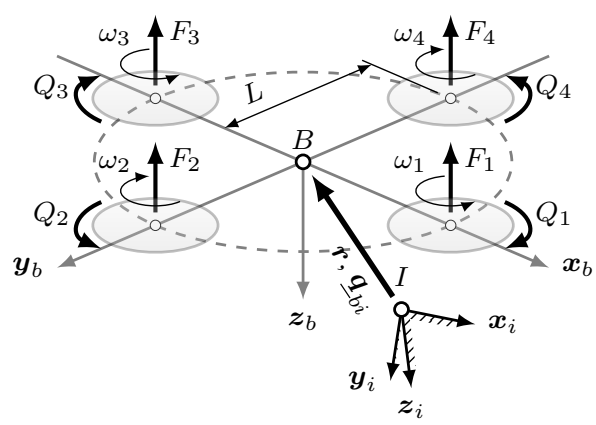

Fig. 2: The considered quadrotor UAV with position $\boldsymbol{r}$ and attitude $\underline{\boldsymbol{q}}_{b i}$ is controlled by four propellers that generate thrusts $F_{i}$ and torques $Q_{i}, i=1 \ldots 4$. The propellers are located in one plane, at distance $L$ from the center of gravity.

\section{B. Dynamics}

The state of a quadrotor in free flight, shown in Fig. 2, can be described by its position in the inertial frame $r=$ $\left[\begin{array}{lll}x & y & z\end{array}\right]^{T}$ and attitude $\underline{\boldsymbol{q}}_{b i}$. The attitude control torques $\boldsymbol{\tau}=$ $\left[\begin{array}{lll}\tau_{x} & \tau_{y} & \tau_{z}\end{array}\right]^{T}$ are provided by the four propellers, together with the total propeller thrust $T$. The time-varying matched model uncertainties and external disturbances are represented in the terms $\boldsymbol{h}_{f}$ and $\boldsymbol{h}_{\tau}$. Its dynamics can then be described by the translational and rotational acceleration $\ddot{\boldsymbol{r}}$ and $\dot{\boldsymbol{\omega}}$, respectively, as

$$
\begin{aligned}
m \ddot{\boldsymbol{r}}(t) & =\left(m g-T(t) \boldsymbol{R}_{b i}^{T}(t)\right) \boldsymbol{z}_{i}+\boldsymbol{h}_{f}(t) \\
\boldsymbol{J} \dot{\boldsymbol{\omega}}(t) & =\boldsymbol{\tau}(t)+\mathcal{S}(\boldsymbol{J} \boldsymbol{\omega}(t)) \boldsymbol{\omega}(t)+\boldsymbol{h}_{\tau}(t)
\end{aligned}
$$

where $m$ and $\boldsymbol{J}$ are the quadrotor mass and moment of inertia, which are assumed constant. Here, $\boldsymbol{z}_{i}=\left[\begin{array}{lll}0 & 0 & 1\end{array}\right]^{T}$ represents the unit vector in the inertial $z$-direction, and $g$ is the acceleration of gravity. Hence, the first term in the position dynamics is the quadrotor weight, and the second term is the total thrust transformed into the inertial frame. The rotation matrix $\boldsymbol{R}_{b i}=\boldsymbol{R}\left(\underline{\boldsymbol{q}}_{b i}\right)$ transforms a vector from frame $I$ to frame $B$. For notational simplicity, timedependency will be assumed throughout the paper, and the suffix $(t)$ dropped where applicable. Note that here we assume that the drag forces and torques can be lumped in the terms $\boldsymbol{h}_{f}$ and $\boldsymbol{h}_{\tau}$, respectively.

The quadrotor is a nonholonomic system, having four control inputs and six degrees of freedom. Therefore, only four states can be independently controlled. In this paper we control the position $\boldsymbol{r}$ and angle about the inertial $z$-axis. We adopt a cascaded controller scheme, depicted in Fig. 3, where we first design a robust attitude tracking controller which will track a desired attitude $\underline{\boldsymbol{q}}_{d i}$. The attitude controller calculates the required torques $\tau$ to track the desired attitude. The position controller generates a virtual force input $f=$ $T \boldsymbol{R}_{d i} \boldsymbol{z}_{i}$ to obtain the desired attitude $\underline{\boldsymbol{q}}_{d i}$ and thrust $T$ from geometric considerations.

A simplified actuation model is used where the quadrotors' four rotors are located in the same plane as the center of gravity, at arm length $L$ in the principal axes directions, and are numbered in the positive $z$-axis sense starting at 1 on the positive $x$-axis, as depicted in Fig. 2. A simplified model 


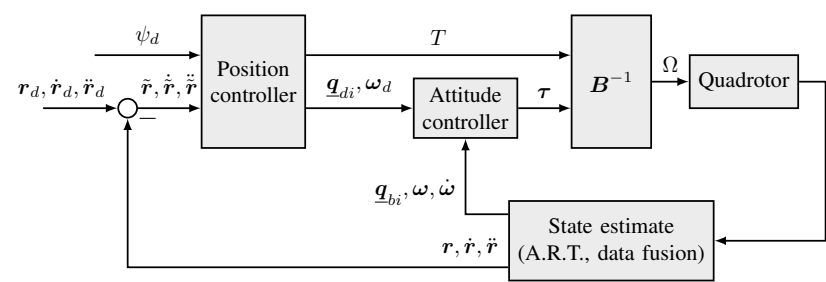

Fig. 3: Block diagram of the complete controller structure

for the rotor thrust $F_{i}=k_{F} \omega_{i}^{2}$ and drag torque $Q_{i}=k_{Q} \omega_{i}^{2}$ of the rotor $i$ is adopted, where $k_{F}$ and $k_{Q}$ are the thrust and torque coefficients, respectively, and drag torque is in the direction opposite of the rotor angular velocity $\omega_{i}$, The control allocation problem can be formulated as $\boldsymbol{u}=\boldsymbol{B} \Omega$, where $\boldsymbol{u}$ are the desired forces and torques in the body frame, $\boldsymbol{B}$ is the control allocation matrix, and $\boldsymbol{\Omega}$ are the squares of the propeller angular velocities. For the quadrotor depicted in Fig. 2 it can be formulated as

$$
\left[\begin{array}{c}
\tau_{x} \\
\tau_{y} \\
\tau_{z} \\
T
\end{array}\right]=\left[\begin{array}{cccc}
0 & -k_{F} L & 0 & k_{F} L \\
k_{F} L & 0 & -k_{F} L & 0 \\
-k_{Q} & k_{Q} & -k_{Q} & k_{Q} \\
k_{F} & k_{F} & k_{F} & k_{F}
\end{array}\right]\left[\begin{array}{c}
\omega_{1}^{2} \\
\omega_{2}^{2} \\
\omega_{3}^{2} \\
\omega_{4}^{2}
\end{array}\right]
$$

where the body $x$ - and $y$ - forces have been omitted as they are always zero. The required angular velocities can be obtained as $\boldsymbol{\Omega}=\boldsymbol{B}^{-1} \boldsymbol{u}$, and for this model, the solution can be obtained analytically.

\section{ATtITUDE TRACKING CONTROL}

\section{A. Attitude error and stabilization}

The attitude error $\underline{\tilde{q}}$ between the desired attitude $\underline{\boldsymbol{q}}_{d i}$ and the body attitude $\underline{\boldsymbol{q}}_{b i}$ is defined geometrically [5], [9], and is equivalent to $\tilde{\boldsymbol{R}}=\boldsymbol{R}(\underline{\tilde{\boldsymbol{q}}})=\boldsymbol{R}_{d i}^{T} \boldsymbol{R}_{b i}$. The error represents the rotation from frame $D$ to frame $B$. Using quaternion multiplication it can be defined as

$$
\begin{aligned}
& \underline{\tilde{\boldsymbol{q}}}=\underline{\boldsymbol{q}}_{d b}=\underline{\boldsymbol{q}}_{d i}^{*} \otimes \underline{\boldsymbol{q}}_{b i} \\
& \underline{\tilde{\boldsymbol{q}}}=\left[\begin{array}{cc}
\tilde{\eta} \\
\tilde{\boldsymbol{\epsilon}}
\end{array}\right]=\left[\begin{array}{cc}
\eta_{d i} & \boldsymbol{\epsilon}_{d i}^{T} \\
-\boldsymbol{\epsilon}_{d i} & \eta_{d i} \boldsymbol{I}_{3 \times 3}-\mathcal{S}\left(\boldsymbol{\epsilon}_{d i}\right)
\end{array}\right]\left[\begin{array}{l}
\eta_{b i} \\
\boldsymbol{\epsilon}_{b i}
\end{array}\right]
\end{aligned}
$$

where $\otimes$ represents quaternion multiplication. The angular velocity error transforms the desired angular velocity from the $D$-frame to the $B$-frame and is defined as

$$
\tilde{\boldsymbol{\omega}}=\boldsymbol{\omega}-\tilde{\boldsymbol{R}}^{T} \boldsymbol{\omega}_{d}, \quad \dot{\tilde{\boldsymbol{\omega}}}=\dot{\boldsymbol{\omega}}-\tilde{\boldsymbol{R}}^{T} \dot{\boldsymbol{\omega}}_{d}-\dot{\tilde{\boldsymbol{R}}}^{T} \boldsymbol{\omega}_{d}
$$

where $\dot{R}=\boldsymbol{R} \mathcal{S}(\boldsymbol{\omega})$ is the time derivative of the rotation matrix.

To better explain quaternion attitude control, let us first consider the attitude stabilization problem without model uncertainties. Consider the Lyapunov function candidate (LFC)

$$
V=\frac{1}{2} \boldsymbol{\omega}^{T} \boldsymbol{J} \boldsymbol{\omega}+2 c H(\tilde{\eta})
$$

where the inertia $\boldsymbol{J}$ is constant, $c$ is positive and $H(\tilde{\eta})$ is the quaternion error function. Because the error is a unit quaternion, the error function must be a Lipschitz function defined on the range $[-1,1]$ and vanish at \pm 1 , since $\tilde{\eta}= \pm 1$ represents aligned $B$ and $D$ frames. Several common choices for $H(\tilde{\eta})$ can be found in [5]. After expansion, the derivative of the LFC is

$$
\dot{V}=\boldsymbol{\omega}^{T}\left[\boldsymbol{\tau}+\mathcal{S}(\boldsymbol{J} \boldsymbol{\omega}) \boldsymbol{\omega}+\boldsymbol{h}_{\tau}\right]-c \boldsymbol{\omega}^{T} \tilde{\boldsymbol{e}}
$$

where $\tilde{\boldsymbol{e}}=-\frac{\partial H}{\partial \tilde{\eta}} \tilde{\boldsymbol{\epsilon}}$ has been introduced. By assuming $\boldsymbol{h}_{\tau}=\mathbf{0}$ and taking the control law $\boldsymbol{\tau}=-\boldsymbol{K}_{v} \boldsymbol{\omega}-c \tilde{\boldsymbol{e}}$ with gain $\boldsymbol{K}_{v} \geq$ $\mathbf{0}_{3 \times 3}$, and using the skew-symmetry property of the Coriolis term in the dynamics, $\dot{V}$ becomes negative semidefinite

$$
\dot{V}=-\boldsymbol{\omega}^{T} \boldsymbol{K}_{v} \boldsymbol{\omega}<0, \quad \forall \boldsymbol{\omega} \neq \mathbf{0}
$$

The equilibrium points depend on the chosen potential function of the quaternion error. Due to the structure of the $\mathrm{SO}(3)$ group, at least two equilibrium points exist In this paper, we choose $H(\tilde{\eta})=\tilde{\boldsymbol{\epsilon}}^{T} \tilde{\boldsymbol{\epsilon}}$, which gives $\tilde{\boldsymbol{e}}=2 \tilde{\eta} \tilde{\boldsymbol{\epsilon}}$ is similar to angle-axis feedback but does not suffer from singularities at zero error. When using this error function, the asymptotically stable equilibrium points are $\tilde{\eta}= \pm 1$, and an unstable equilibrium point exists at $\tilde{\eta}=0$. The function has been chosen because it is continuous and is stable at both signs of $\tilde{\eta}$.

\section{B. Robust attitude tracking control}

Define the desired attitude error dynamics to be

$$
\dot{\tilde{\boldsymbol{\omega}}}+\boldsymbol{K}_{v} \dot{\tilde{\boldsymbol{e}}}+c \tilde{\boldsymbol{e}}=\mathbf{0}
$$

where instead of the angular velocity error, we have taken the time derivative of the geometric attitude error. For control design we take an ideal model without disturbances to be followed

$$
\boldsymbol{J}_{0} \dot{\boldsymbol{\omega}}_{0}=\boldsymbol{\tau}_{0}+\mathcal{S}\left(\boldsymbol{J}_{0} \boldsymbol{\omega}_{0}\right) \boldsymbol{\omega}_{0}
$$

In integral sliding mode controller design, the tracking (sliding) variable is expanded by a model-based integral term $z$ which is designed such that sliding mode starts from the first time instant. By defining the sliding variable $s$ to be

$$
\boldsymbol{s}=\boldsymbol{s}_{0}+\boldsymbol{z}, \quad \boldsymbol{s}_{0}=\tilde{\boldsymbol{\omega}}+\boldsymbol{K}_{v} \tilde{\boldsymbol{e}}
$$

from the sliding condition $\dot{s}=\mathbf{0}$, one obtains

$$
\dot{\boldsymbol{z}}=-\dot{\boldsymbol{s}}_{0}=-\dot{\tilde{\boldsymbol{\omega}}}_{0}-\boldsymbol{K}_{v} \dot{\tilde{\boldsymbol{e}}}, \quad \boldsymbol{z}(0)=-\boldsymbol{s}_{0}(0)
$$

where the ideal error dynamics $\dot{\tilde{\omega}}_{0}$ is obtained from the nominal model (9), using (6). The actual error dynamics can be obtained from (3).

$$
\begin{aligned}
& \dot{\tilde{\boldsymbol{\omega}}}_{0}=\boldsymbol{J}_{0}^{-1} \boldsymbol{\tau}_{0}+\boldsymbol{J}_{0}^{-1} \mathcal{S}\left(\boldsymbol{J}_{0} \boldsymbol{\omega}\right) \boldsymbol{\omega}-\tilde{\boldsymbol{R}}^{T} \dot{\boldsymbol{\omega}}_{d}-\dot{\tilde{\boldsymbol{R}}}^{T} \boldsymbol{\omega}_{d} \\
& \dot{\tilde{\boldsymbol{\omega}}}=\boldsymbol{J}^{-1} \boldsymbol{\tau}+\boldsymbol{J}^{-1} \mathcal{S}(\boldsymbol{J} \boldsymbol{\omega}) \boldsymbol{\omega}+\boldsymbol{J}^{-1} \boldsymbol{h}_{\tau}-\tilde{\boldsymbol{R}}^{T} \dot{\boldsymbol{\omega}}_{d}-\dot{\tilde{\boldsymbol{R}}}^{T} \boldsymbol{\omega}_{d}
\end{aligned}
$$

The control law $\tau$ consists of a nominal control $\tau_{0}$ and a robust control $\tau_{1}$. The nominal control law is obtained such that the nominal model (9) follows the desired error dynamics (8). The robust control is determined from the stability analysis.

$$
\begin{aligned}
\boldsymbol{\tau} & =\boldsymbol{\tau}_{0}+\boldsymbol{\tau}_{1} \\
\boldsymbol{\tau}_{0} & =\boldsymbol{J}_{0}\left(\tilde{\boldsymbol{R}}^{T} \dot{\boldsymbol{\omega}}_{d}+\dot{\tilde{\boldsymbol{R}}}^{T} \boldsymbol{\omega}_{d}-\boldsymbol{K}_{v} \dot{\tilde{\boldsymbol{e}}}-c \tilde{\boldsymbol{e}}\right)-\mathcal{S}\left(\boldsymbol{J}_{0} \boldsymbol{\omega}\right) \boldsymbol{\omega}
\end{aligned}
$$


The derivative of the sliding variable (10) is then

$$
\begin{aligned}
\dot{\boldsymbol{s}} & =\dot{\boldsymbol{s}}_{0}+\dot{z}=\boldsymbol{\zeta}_{1}+\boldsymbol{\zeta}_{2} \boldsymbol{\tau}_{0}+\boldsymbol{J}^{-1} \boldsymbol{\tau}_{1}+\boldsymbol{J}^{-1} \boldsymbol{h}_{\tau} \\
\boldsymbol{\zeta}_{1} & =\boldsymbol{J}^{-1} \mathcal{S}(\boldsymbol{J} \boldsymbol{\omega}) \boldsymbol{\omega}-\boldsymbol{J}_{0}^{-1} \mathcal{S}\left(\boldsymbol{J}_{0} \boldsymbol{\omega}\right) \boldsymbol{\omega} \\
\boldsymbol{\zeta}_{2} & =\boldsymbol{J}^{-1}-\boldsymbol{J}_{0}^{-1}
\end{aligned}
$$

The disturbance $\boldsymbol{h}_{\tau}$ can be approximated from the discretetime formulation of the dynamics (3). We assume a small sampling time $t_{s} \ll 1$, so that $\boldsymbol{h}_{\tau, k}$ for $t=k t_{s}$ can be considered constant between time steps:

$$
\boldsymbol{h}_{\tau, k} \approx \boldsymbol{h}_{\tau, k-1}=\boldsymbol{J}_{0} \dot{\boldsymbol{\omega}}_{k-1}-\boldsymbol{\tau}_{k-1}-\mathcal{S}\left(\boldsymbol{J} \boldsymbol{\omega}_{k-1}\right) \boldsymbol{\omega}_{k-1}
$$

This measurement will include both modeling errors and external disturbance. If a more accurate model of external forces are available, e.g. a drag model, it can be directly included in (15) to improve the estimation. The acceleration signal is typically noisy, especially if it comes from differentiating a velocity measurement. To improve robustness and reduce sensitivity to noise, the disturbance estimation is lowpass-filtered. Let us define the estimation error as

$$
\tilde{\boldsymbol{h}}_{\tau}=\boldsymbol{h}_{\tau}-\hat{\boldsymbol{h}}_{\tau}
$$

Next we take the Lyapunov function

$$
V=\frac{1}{2} \boldsymbol{s}^{T} \boldsymbol{J} \boldsymbol{s}+\frac{1}{2} \tilde{\boldsymbol{h}}_{\tau}^{T} \boldsymbol{\Gamma}^{-1} \tilde{\boldsymbol{h}}_{\tau}
$$

whose derivative is

$$
\dot{V}=\boldsymbol{s}^{T} \boldsymbol{J}\left(\boldsymbol{\zeta}_{1}+\boldsymbol{\zeta}_{2} \boldsymbol{\tau}_{0}\right)+\boldsymbol{s}^{T} \boldsymbol{\tau}_{1}+\boldsymbol{s}^{T} \boldsymbol{h}_{\tau}+\tilde{\boldsymbol{h}}_{\tau}^{T} \boldsymbol{\Gamma}^{-1}\left(\dot{\boldsymbol{h}}_{\tau}-\dot{\hat{\boldsymbol{h}}}_{\tau}\right)
$$

By choosing the robust control law $\tau_{1}$ and disturbance estimation dynamics to be

$$
\boldsymbol{\tau}_{1}=-\boldsymbol{K}_{w} \operatorname{sgn}(\boldsymbol{s})-\hat{\boldsymbol{h}}_{\tau}, \quad \dot{\hat{\boldsymbol{h}}}_{\tau}=\boldsymbol{\Gamma} \tilde{\boldsymbol{h}}_{\tau}+\rho \operatorname{sgn}\left(\tilde{\boldsymbol{h}}_{\tau}\right)
$$

the derivative of the Lyapunov function becomes

$$
\begin{aligned}
\dot{V} & =\boldsymbol{s}^{T} \boldsymbol{J}\left(\boldsymbol{\zeta}_{1}+\boldsymbol{\zeta}_{2} \boldsymbol{\tau}_{0}\right)-\boldsymbol{s}^{T} \boldsymbol{K}_{w} \operatorname{sgn}(\boldsymbol{s})-\boldsymbol{s}^{T} \tilde{\boldsymbol{h}}_{\tau} \\
& -\tilde{\boldsymbol{h}}_{\tau}^{T} \tilde{\boldsymbol{h}}_{\tau}+\tilde{\boldsymbol{h}}_{\tau}^{T} \boldsymbol{\Gamma}^{-1}\left(\dot{\boldsymbol{h}}_{\tau}-\rho \operatorname{sign}\left(\tilde{\boldsymbol{h}}_{\tau}\right)\right)
\end{aligned}
$$

For (20) to be negative definite, it must hold that

$$
\begin{array}{r}
\boldsymbol{K}_{w}>\frac{1}{\gamma}\left\|\boldsymbol{\zeta}_{1}+\boldsymbol{\zeta}_{2} \boldsymbol{\tau}_{0}+\boldsymbol{J}^{-1} \tilde{\boldsymbol{h}}_{\tau}\right\|, \quad \gamma<\lambda_{\min }\left(\boldsymbol{J}^{-1}\right) \\
\rho>\sup \left\|\dot{\boldsymbol{h}}_{\tau}\right\|
\end{array}
$$

where it is assumed that $\boldsymbol{h}_{\tau}$ is Lipschitzian. It can be seen that the sliding gain $\boldsymbol{K}_{w}$ depends on the modeling error $\boldsymbol{\zeta}_{1}+\boldsymbol{\zeta}_{2} \boldsymbol{\tau}_{0}$ as well as the disturbance estimation error $\tilde{\boldsymbol{h}}_{\tau}$. If the disturbance had not been estimated, the gain would also have to be larger than the maximum amplitude of the external disturbance, as well as the modeling errors. Thus, by incorporating an explicit disturbance estimator into the controller, the sliding gain can be smaller, which leads to improved robustness. The gain $\rho$ in the disturbance estimator compensates for the rate of change of the disturbance. If $\rho$ is taken zero, the sliding gain has to be higher in order to compensate the disturbance component $\dot{\boldsymbol{h}}_{\tau}$. The equivalent control, or averaged motion, of the sliding mode term is

$$
\boldsymbol{\tau}_{1, \mathrm{eq}}=\boldsymbol{J}\left(\boldsymbol{\zeta}_{1}+\boldsymbol{\zeta}_{2} \boldsymbol{\tau}_{0}\right)+\tilde{\boldsymbol{h}}_{\tau}
$$

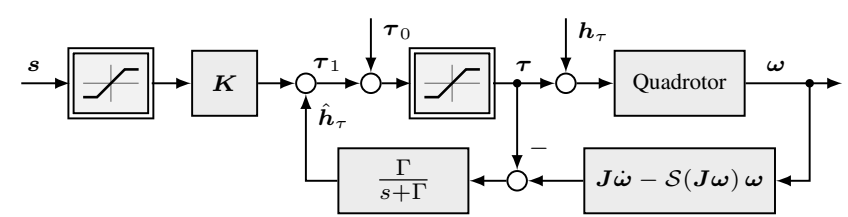

Fig. 4: Block diagram of the attitude controller and disturbance observer.

Since using a signum function in the control leads to chattering, we adopt a boundary-layer approach, which is equivalent to a lowpass-filtered signal of the signum function. By taking

$$
\boldsymbol{\tau}_{1}=-\boldsymbol{K}_{w} \operatorname{sat}(s / \varepsilon)-\hat{\boldsymbol{h}}_{\tau}
$$

with a small constant $\varepsilon>0$, the system will behave as in (20) outside the boundary layer. The system will therefore not converge asymptotically to the tracking variable, but to an $\varepsilon$-vicinity thereof. Inside the boundary layer we can define an equivalent gain $\boldsymbol{K}=\boldsymbol{K}_{w} / \varepsilon$ and set $\rho=0$ to obtain

$$
\begin{aligned}
\dot{V} & =\boldsymbol{s}^{T} \boldsymbol{J}\left(\boldsymbol{\zeta}_{1}+\boldsymbol{\zeta}_{2} \boldsymbol{\tau}_{0}\right)+\tilde{\boldsymbol{h}}_{\tau}^{T} \boldsymbol{\Gamma}^{-1} \dot{\boldsymbol{h}}_{\tau}-W \\
W & =\boldsymbol{s}^{T} \boldsymbol{K} \boldsymbol{s}+\boldsymbol{s}^{T} \tilde{\boldsymbol{h}}_{\tau}+\tilde{\boldsymbol{h}}_{\tau}^{T} \boldsymbol{\Gamma} \tilde{\boldsymbol{h}}_{\tau} \\
& \geq \lambda_{\min }\{\boldsymbol{K}\}\|\boldsymbol{s}\|^{2}+\|\boldsymbol{s}\|\left\|\tilde{\boldsymbol{h}}_{\tau}\right\|+\lambda_{\min }\{\boldsymbol{\Gamma}\}\left\|\tilde{\boldsymbol{h}}_{\tau}\right\|^{2}
\end{aligned}
$$

and the condition for its positive definiteness of $W$ is

$$
4 \lambda_{\min }\{\boldsymbol{K}\} \lambda_{\min }\{\boldsymbol{\Gamma}\}>1
$$

hence the system must be sufficiently damped inside the boundary layer to compensate the estimation error dynamics. An upper limit on $\boldsymbol{K}$ is imposed by the propeller dynamics and sensor noise. The Lyapunov function inside the boundary layer will not be negative definite, but will be dominated by the model and estimation errors. The error dynamics inside the boundary layer in case of $\boldsymbol{J} \neq \boldsymbol{J}_{0}$ can be obtained as

$$
\begin{aligned}
\dot{\tilde{\boldsymbol{\omega}}}+\boldsymbol{J}^{-1} \boldsymbol{J}_{0} \boldsymbol{K}_{v} \tilde{\boldsymbol{\omega}}+\boldsymbol{J}^{-1} \boldsymbol{J}_{0} c \tilde{\boldsymbol{e}}=\boldsymbol{\zeta}_{1}-\boldsymbol{J}^{-1} \tilde{\boldsymbol{h}}_{\tau} \\
\dot{\hat{\boldsymbol{h}}}_{\tau}+\boldsymbol{\Gamma} \hat{\boldsymbol{h}}_{\tau}=\boldsymbol{\Gamma} \tilde{\boldsymbol{h}}_{\tau}
\end{aligned}
$$

which shows that the error dynamics inside the boundary layer is excited by the disturbance estimation error.

The boundary-layer integral sliding mode controller behaves like a saturated PID controller, therefore an antiwindup method must be applied, as shown in [29]. Therein, during saturation of the sliding mode term in $\tau_{1}$, the sliding surface is reset such that $z=-s_{0}$ and $\dot{z}=\mathbf{0}$. Furthermore, saturated control inputs are used for disturbance observation, as depicted in Fig. 4.

\section{POSITION TRACKING CONTROL}

The position controller is designed to track a desired position $\boldsymbol{r}_{d}=\left[\begin{array}{lll}x_{d} & y_{d} & z_{d}\end{array}\right]^{T}$, velocity $\dot{\boldsymbol{r}}_{d}$ and acceleration $\ddot{\boldsymbol{r}}_{d}$. The angle $\psi$ about the inertial $z$-axis remains free to be controlled. This is achieved by designing a controller in the inertial frame which calculates an inertial control force. The force is then used to generate an attitude and thrust reference for the underlying attitude controller. 


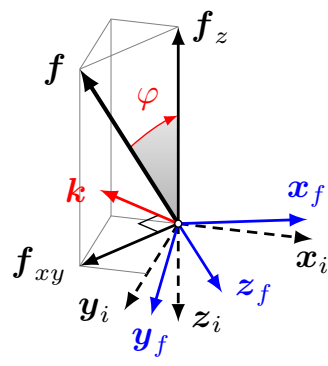

(a) Thrust transformation $\underline{\boldsymbol{q}}_{f}$

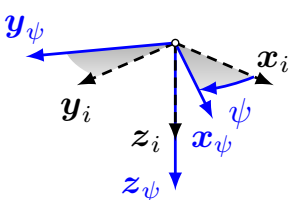

(b) Yaw transformation $\underline{\boldsymbol{q}}_{\psi}$
Fig. 5: Transformations involved in creating an attitude reference from the virtual control force $f$.

\section{A. Virtual control force}

The design closely follows that of the attitude controller, therefore most details are omitted. The controller calculates a required force in the inertial frame based on the desired error dynamics

$$
\ddot{\tilde{\boldsymbol{r}}}+\boldsymbol{K}_{v, p} \dot{\tilde{\boldsymbol{r}}}+\boldsymbol{K}_{p, p} \tilde{\boldsymbol{r}}=\mathbf{0}
$$

where $\tilde{\boldsymbol{r}}=\boldsymbol{r}-\boldsymbol{r}_{d}$ is the position error. We follow the ideal disturbance-free dynamics based on (3)

$$
m_{0} \ddot{\boldsymbol{r}}_{0}=\left(m_{0} g-T \boldsymbol{R}_{b i}\right) \boldsymbol{z}_{i}
$$

where $m_{0}$ is the nominal mass. A quaternion reference attitude is then generated that aligns the thrust vector with the desired control force. Equivalent to the attitude controller, the position control input $f=f_{0}+f_{1}$ consists of a nominal control $f_{0}$, based on (28) and (27), and a robust control $f_{1}$ such that

$$
\begin{aligned}
& \boldsymbol{f}_{0}=m_{0}\left(\ddot{\boldsymbol{r}}_{d}-\boldsymbol{K}_{v, p} \dot{\tilde{\boldsymbol{r}}}-\boldsymbol{K}_{p, p} \tilde{\boldsymbol{r}}\right) \\
& \boldsymbol{f}_{1}=-\hat{\boldsymbol{h}}_{f}-\boldsymbol{K}_{f} \operatorname{sat}\left(\boldsymbol{s}_{p} / \boldsymbol{\varepsilon}_{p}\right)
\end{aligned}
$$

where $\ddot{\boldsymbol{r}}_{d}$ includes the gravity compensation term. The disturbance $\boldsymbol{h}_{f}$ in the inertial frame is obtained by

$$
\dot{\hat{\boldsymbol{h}}}_{f}=\boldsymbol{\Gamma}_{f}\left(m_{0} \ddot{\boldsymbol{r}}-m_{0} g \boldsymbol{z}_{i}-\boldsymbol{f}-\hat{\boldsymbol{h}}_{f}\right)
$$

By using the same integral sliding mode design process as in the attitude controller, the sliding variable is obtained to be

$$
\boldsymbol{s}_{p}=\dot{\tilde{\boldsymbol{r}}}+\boldsymbol{K}_{v, p} \tilde{\boldsymbol{r}}+\int \boldsymbol{K}_{p, p} \tilde{\boldsymbol{r}} \mathrm{d} t-\dot{\tilde{\boldsymbol{r}}}(0)-\boldsymbol{K}_{v, p} \tilde{\boldsymbol{r}}(0)
$$

Hence, the controller behaves locally as a PID controller. The thrust is equal to the norm of the desired force, i.e. $T=\|\boldsymbol{f}\|$.

\section{B. Attitude reference generation}

The attitude reference can be obtained from $f$ through two transformations, depicted in Fig. 5. The thrust transformation $\underline{\boldsymbol{q}}_{f}$ aligns the $z_{f}$-axis to the desired thrust vector, and is obtained from an angle-axis representation. The yaw transformation $\underline{\boldsymbol{q}}_{\psi}$ rotates about the inertial $z$-axis by angle $\psi$. The thrust vector points in the negative $\boldsymbol{z}_{i}$ direction in hover, so a rotation axis $\boldsymbol{k}$ can be obtained as the cross product between the desired inertial force and the negative $\boldsymbol{z}_{i}$ vector. The transformation between axes $\boldsymbol{z}_{i}$ and $\boldsymbol{z}_{f}$ can be obtained by normalizing the non-unit quaternion $\underline{\boldsymbol{q}}_{f}=\left[\eta_{f} \boldsymbol{\epsilon}_{f}\right]^{T}$

$$
\eta_{f}=-\boldsymbol{z}_{i}^{T} \boldsymbol{f}+\sqrt{1+\boldsymbol{f}^{T} \boldsymbol{f}}, \quad \boldsymbol{\epsilon}_{f}=-\boldsymbol{z}_{i} \times \boldsymbol{f}
$$

The reference quaternion $\underline{\boldsymbol{q}}_{d i}$ from the position controller is then obtained by transforming the yaw coordinate system by the thrust transform:

$$
\underline{\boldsymbol{q}}_{d i}=\underline{\boldsymbol{q}}_{f}^{0} \otimes \underline{\boldsymbol{q}}_{\psi}, \quad \underline{\boldsymbol{q}}_{\psi}=\left[\cos \frac{\psi}{2}, 0,0, \sin \frac{\psi}{2}\right]^{T}
$$

The transformation is free of singularities. Here, the yaw transform represents the angle about the inertial $z$-axis, and not the Euler yaw angle.

Also commanding an angular velocity improves attitude tracking performance. The angular velocity command can be generated by discretizing the kinematics of the rotation matrix and calculating delta rotations of the position controller between two time steps. The angular velocity at time step $k$ is then

$$
\boldsymbol{\omega}_{d, k}=\vee\left(\boldsymbol{R}_{b i, k-1}^{T} \boldsymbol{R}_{b i, k}\right) t_{s}^{-1}
$$

where $t_{s}$ is the sampling time of the position controller, and $\checkmark(\cdot)$ is the inverse of the skew-symmetric matrix operator, which extracts a vector from the matrix $\mathcal{S}(\cdot)$. In this way, angular velocity is generated from the feedback signal, rather than the feedforward signal from a precomputed trajectory.

\section{RESULtS}

In this section, we first show the behavior of the controller with disturbance observation when model uncertainties are applied in simulation. The transient response is compared to a PID controller and influence of the integral term is shown. Three sets of experiments are presented, where four controllers are compared - PID, Adaptive Integral Backstepping Controller (AIBC), Integral Sliding Mode with Disturbance Observation (ISM+DO) and PD with disturbance observation (PD+DO). First, we compare hovering performance with and without turbulent wind influence across position and attitude controllers. Second, we show trajectory tracking between waypoints, where large angles must be applied. Lastly, we show behavior of the disturbance observation method when a constant force in the inertial frame is applied, and compare the response with the AIBC. For presentation clarity, we show the ZYX Euler angles, while quaternions are used for control.

For a fair comparison, all controllers have been tuned to have the same local closed-loop gains as a standard PID controller $f_{\mathrm{PID}, x}=m_{0}\left(\ddot{x}_{d}-K_{v, x} \dot{\tilde{x}}-K_{p, x} \tilde{x}-K_{i, x} \int \tilde{x} \mathrm{~d} \tau\right)$ with gains $\boldsymbol{K}_{v, p}=2 \omega_{c}, \boldsymbol{K}_{v, p}=\omega_{c}^{2}, \boldsymbol{K}_{i, p}=\frac{1}{4} \omega_{c}$. This applies for the sliding mode boundary layer and AIBC controller. Disturbance observers were tuned separately. The AIBC position controller was implemented as three decoupled controllers of the form from [18], [19] as

$f_{x}=m_{0}\left(\ddot{x}_{d}-\left(1-c_{1}^{2}+\lambda\right) \tilde{x}-\left(c_{1}+c_{2}\right) \dot{\tilde{x}}+c_{1} \lambda \xi_{x}-\hat{h}_{x}\right)$ 


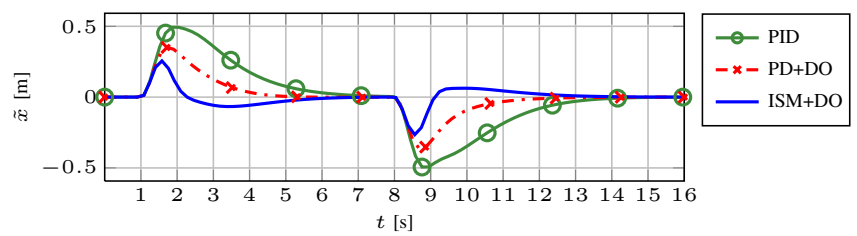

Fig. 6: Simulative comparison of position controller errors for setpoint control and disturbance. A constant force disturbance of $2 \mathrm{~N}$ is applied in the inertial $x$-direction between $1 \mathrm{~s}$ and $8 \mathrm{~s}$. The controller parameters for the $x$-axis are $m_{0}=m=0.5 \mathrm{~kg}$, $\omega_{c, x}=1.5 \mathrm{rad} / \mathrm{s}, K_{s f, x}=1.5 m_{0}, \varepsilon=1, \Gamma_{f, x}=5$.

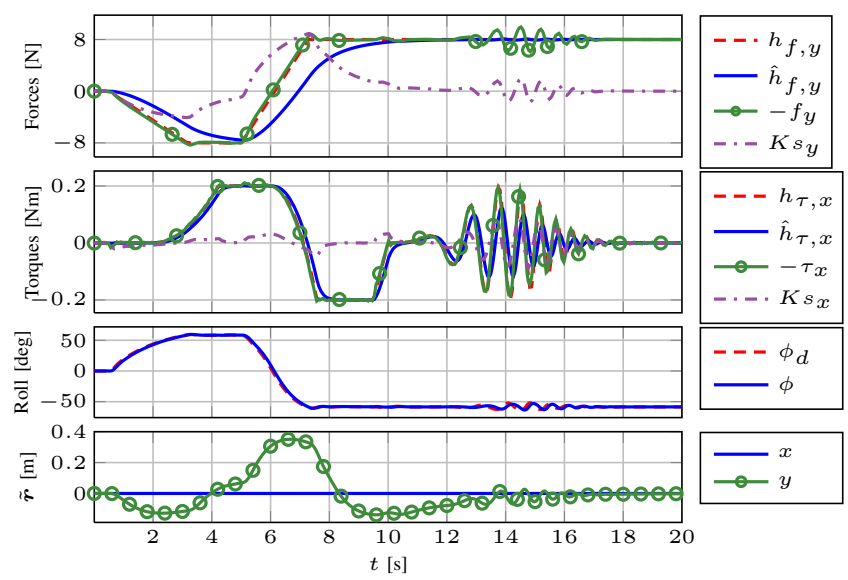

Fig. 7: Estimation and compensation of time-varying disturbances in simulation, using the ISM+DO method. The quadrotor is commanded to hover in place while the disturbances are applied on the roll axis and in the inertial $y$-direction. The disturbance compensation is faster due to ISM, and the tracking variable $s$ (term $K s_{y}$ ) converges to zero when the disturbance is constant.

where $\xi_{x}=\int \tilde{x} \mathrm{~d} \tau$ for the $x$-axis and equivalent for other axes, with the disturbance estimation as $\dot{\hat{h}}_{x}=\gamma \dot{e}$. Since the mass is constant and is not adapted in other controllers, the adaptation has not been implemented for the AIBC.

\section{A. Simulation results}

The simulation model includes sensor noise, propeller modeling and actuator discretization. Angular accelerations are obtained through finite-difference differentiation. Fig. 6 shows the step position disturbance response for different controllers, which is applied between $1 \mathrm{~s}$ and $8 \mathrm{~s}$, with an ISM+DO attitude controller. The PD+DO approach shows faster convergence than the PID controller. The combined ISM+DO scheme inside the boundary layer has a faster response and a smaller absolute error, however the transient has an undershoot due to integrator influence.

Next we show the response to time-varying disturbances in Fig. 7. The quadrotor is commanded to hover at a constant position, while time-varying disturbances are applied, as a torque about the $x$-axis (roll), and a force in the inertial $y$ axis. The force is counteracted by changing the roll angle, and is thereby coupled with the torque disturbance. It can be seen that the disturbance is counteracted very closely despite being time-varying. The slow component is identified by the acceleration-based disturbance estimator, while the faster component is counteracted through the integral sliding mode term $K \boldsymbol{s}$. A chirp torque disturbance with varying amplitude can be compensated, even though the lowpass disturbance estimate has a considerable phase delay. The system is in the boundary layer, and the sliding variable $s$ goes to zero once the constant disturbance is counteracted by the disturbance estimate. Position and attitude are of course coupled, as a change in the attitude due to the disturbance causes a change in position, so the quadrotor must fly back to the hover position. Note that here the disturbance amplitude is larger than the quadrotor weight, so the roll angle must be held at 60 degrees in hover.

\section{B. Experimental validation}

The experiments are carried out using an AscTec Hummingbird that runs the quaternion attitude controller with disturbance observer onboard at $1 \mathrm{kHz}$. A strapdown algorithm integrates the onboard gyros to obtain the attitude estimate that is used for the control. The attitude drift is corrected from motion tracking measurements. Position and attitude measurement are provided by an A.R.T. motion tracking system at $60 \mathrm{~Hz}$. The position controller runs in Simulink, and sends attitude and thrust reference commands to the quadrotor via a wireless XBee link. The angular acceleration is obtained onboard by numerically differentiating the gyro signals. Translational velocity and acceleration are obtained by differentiating and filtering raw position measurements from the motion tracking system, hence a small delay is introduced.

We first investigate the influence of a disturbance observer in hover conditions. All experiments have been carried out with the same hardware and under same conditions. Since it is common to use a PD attitude controller on the Hummingbird platform, we compare the quaternion controller in PD form and with disturbance observation. The quadrotor parameters are not ideally known, so modeling errors exists. Therefore, as no external disturbances are present, these will dominate the error dynamics. Fig. 8.a. shows box plots for the four position controllers and attitude controllers with and without disturbance observation. It can be seen that only using a disturbance observer in the attitude controller improves performance in the horizontal plane. It does significantly not influence altitude control. In hover, the ISM controller is always inside the boundary layer, so the integral term adds a low frequency component that spreads the error distribution when combined with the disturbance observer.

Next, we analyse the influence of turbulent wind on hovering performance. The setup is depicted in Fig. 1.a. The fan generates a turbulent wind stream with a velocity of $3 \mathrm{~m} / \mathrm{s}$, resulting in a $\sim 15^{\circ}$ hover. Fig. 8.b. shows very consistent performance of the $\mathrm{PD}+\mathrm{DO}$ position control approach when combined with the attitude disturbance observer. It can be seen that the PD attitude controller performs much worse in turbulent conditions. It can be concluded that the position controller performance can be significantly improved in turbulent wind conditions by simply adding a disturbance observer to the attitude controller. Adding a disturbance observer to the position control loop further improves per- 
PD+DO att. controller

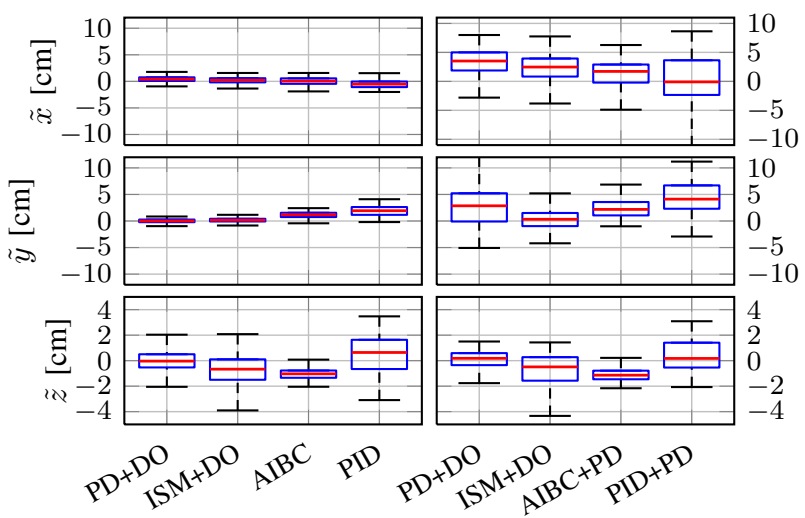

(a) Hovering without wind influence
PD+DO att. controller $\quad$ PD att. controller

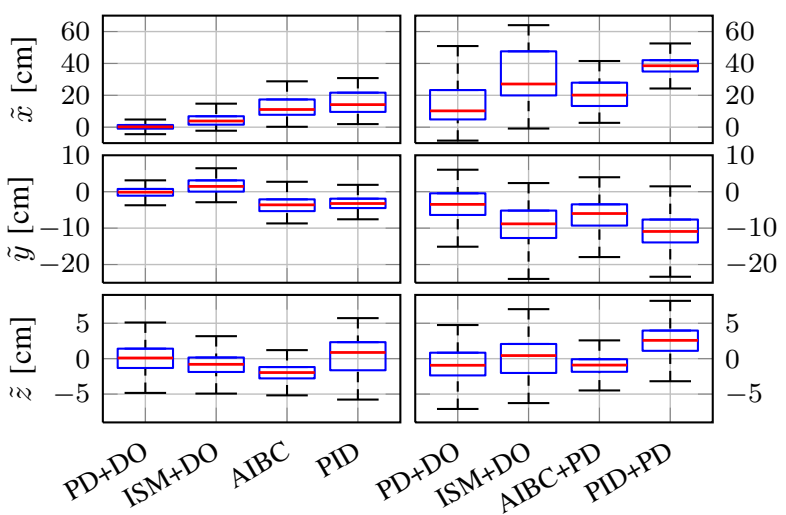

(b) Hovering in $3 \mathrm{~m} / \mathrm{s}$ turbulent wind stream

Fig. 8: Hovering performance using different position and attitude controllers. Controller parameters were constant through the experiments. The attitude controller was tuned as $\omega_{c, a}=12 \mathrm{rad} / \mathrm{s}$ and $\Gamma_{a}=8$. All position controllers had $\omega_{c, p}=1.5 \mathrm{rad} / \mathrm{s}$, and the mass was constant at $m=m_{0}=0.63 \mathrm{~kg}$. Position DO parameters were $\boldsymbol{\Gamma}_{p}=\operatorname{diag}\left\{\left[\begin{array}{lll}3 & 3 & 1.5\end{array}\right]\right\}$ and $\operatorname{AIBC} \boldsymbol{\gamma}=\operatorname{diag}\left\{\left[\begin{array}{lll}4 & 4 & 2\end{array}\right]\right\}$.
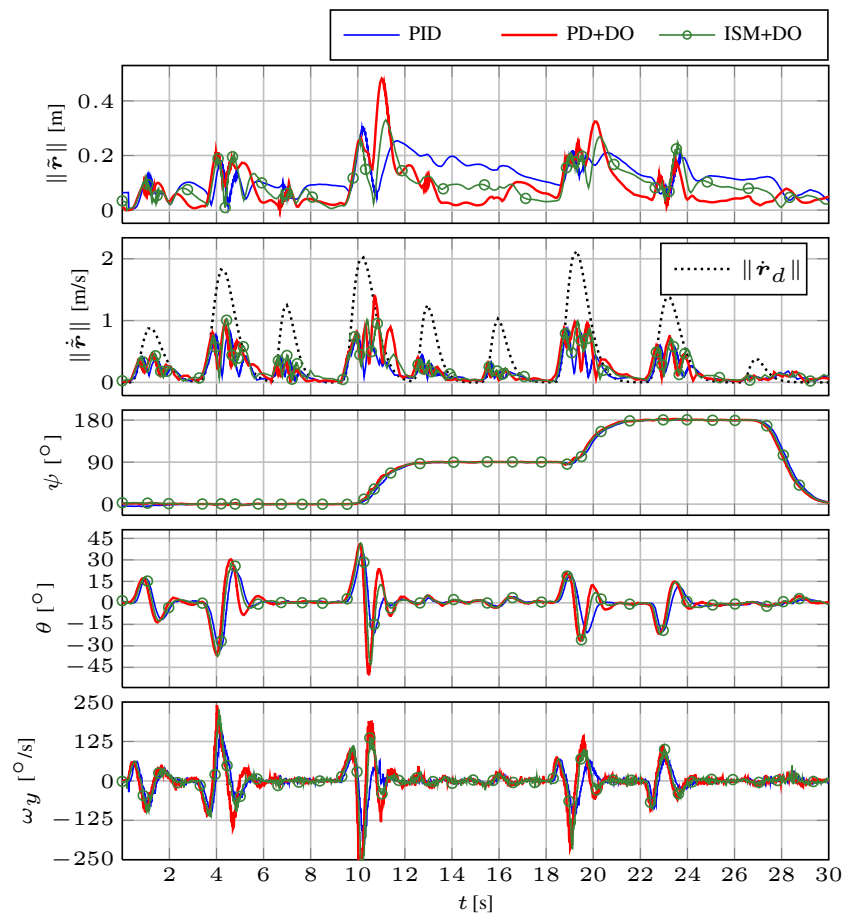

Fig. 9: Position and velocity tracking during aggressive waypoint trajectory tracking. The maximum commanded velocity was $2 \mathrm{~m} / \mathrm{s}$. Due to overshoot, the max. reached velocity was $3 \mathrm{~m} / \mathrm{s}$. The roll and pitch angles reach up to $50^{\circ}$, and the commanded angular velocity reaches the limit of $250^{\circ}$ s. Yaw tracking remains good throughout the flights. The roll angle and angular velocity are not shown.

formance. However, the ISM integral term does not further improve the hovering performance.

Fig. 9 shows position and velocity tracking errors for a trajectory tracking experiment. The velocity and acceleration were generated by filtering the position between waypoints. Here, the maximal reference velocity was about $2 \mathrm{~m} / \mathrm{s}$. The ISM+DO controller shows fast and very oscillatory behavior. It can be seen that the PD+DO controller shows the fastest

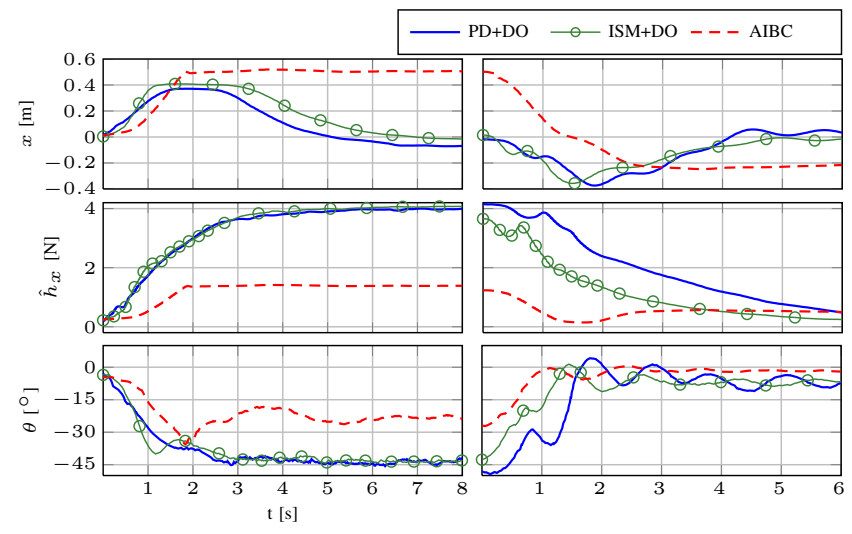

Fig. 10: Position, pitch and disturbance estimate in the suspended weight experiment with PD+DO (-), ISM+DO (-) and AIBC $(--)$ controllers. The left column shows transients when the force is applied to the quadrotor, and the right column when it is released. For the DO-based approaches, the quadrotor hovers at about $45^{\circ}$ in equilibrium. The AIBC does not correctly identify the external force, as the weight falls to the ground. Position DO parameters were $\boldsymbol{\Gamma}_{p}=\operatorname{diag}\left\{\left[\begin{array}{llll}7 & 1.5 & 1.5\end{array}\right]\right\}$ and $\mathrm{AIBC} \boldsymbol{\gamma}=\operatorname{diag}\left\{\left[\begin{array}{lll}8 & 3 & 2\end{array}\right]\right\}$.

convergence of the position error, however it also shows large velocity overshoot. This is due to lumping the unmodeled drag force in the disturbance. Here, performance can be improved by modeling the velocity-dependent drag forces. The obtained pitch angle and commanded angular velocity show that the angles reach $45^{\circ}$, The onboard gyros are rated to $300^{\circ}$ s, so the commanded signal is limited to avoid sensor saturation. This leads to lower tracking performance in periods of high acceleration.

Lastly, we compare the transient response to a step disturbance in the inertial $x$-direction. The setup is shown in Fig. 1.b. A weight of $6 \mathrm{~N}(610 \mathrm{~g})$ is suspended and bound to the quadrotor with a string. The quadrotor, with a mass of $630 \mathrm{~g}$, is then commanded to hover and the weight is dropped. This produces a constant horizontal force in the $x$-direction on the quadrotor. Fig. 10 shows that the 
DO identifies the weight quickly. The quantitative error indicates errors in modeling of the quadrotor thrust. The hover position is reached quickly. The ISM+DO approach shows faster convergence of the error to zero, as shown in simulations. In equilibrium, the quadrotor hovers at approx. $45^{\circ}$. Importantly, releasing the weight does not cause an overshoot. The velocity-based disturbance observation of the AIBC does not correctly identify this disturbance, since the weight falls to the ground and the quadrotor velocity reaches zero. Therefore, the disturbance estimate converges to a constant value. The integral term is too slow to drive the error to zero. Hence, the DO-based approach is well suited for applications where relatively large external forces are present because these are estimated directly from the acceleration.

\section{CONCLUSION}

We have developed a geometric attitude controller based on quaternion feedback that is robust to external disturbances and modeling errors. We have investigated the combination of acceleration-based DO and integral sliding mode (ISM+DO). It is shown through experiments that this approach can improve performance of all position controller. Our position controller generates reference quaternions in a singularity-free way.

We have compared the presented approach to PID and AIBC controllers through extensive experiments on a quadrotor platform. The results show that the presented approach can improve position controller performance in all conditions despite using a very simple quadrotor model. We also show compensation of a large external force, where the quadrotor hovers well outside of standard hover conditions. The integral backstepping controller fails in this experiment. We have found the presented controllers straightforward to tune, as the number of parameters is small. Position tracking performance can be improved by including a velocity-dependent drag model in the controller.

In the future we will investigate combining the presented controller with on-line parameter estimation. A more accurate system model would result in improved performance of the controller. Furthermore, combining the disturbance observation method with an external force-torque sensor would allow the decoupling of external forces and model errors when the UAV is interacting with the environment, thus broadening its range of applications.

\section{ACKNOWLEDGEMENTS}

The authors would like to thank Josip Kasać, Korbinian Schmid and Felix Ruess for helpful discussions.

\section{REFERENCES}

[1] M. Nakao, K. Ohnishi, and K. Miyachi, "A robust decentralized joint control based on interference estimation," in ICRA '87, vol. 4, 1987, pp. 326-331.

[2] K. Youcef-Toumi and O. Ito, "A Time Delay Controller for Systems with Unknown Dynamics," in ACC '88, 1988, p. 904.

[3] V. Utkin and J. Shi, "Integral sliding mode in systems operating under uncertainty conditions," in CDC '96, Kobe, Japan, December 1996.

[4] J. F. Guerrero-Castellanos, A. Hably, N. Marchand, and S. Lesecq, "Bounded attitude stabilization: Application on four-rotor helicopter," in ICRA '07, 2007, pp. 730-735.
[5] O.-E. Fjellstad, "Control of Unmanned Underwater Vehicles in Six Degrees of Freedom - A Quaternion Feedback Approach," Ph.D. dissertation, University of Trondheim, 1994.

[6] G. M. Hoffmann, S. L. Wasl, and C. J. Tomlin, "Quadrotor helicopter trajectory tracking control," in AIAA GNC, 2008.

[7] I. Cowling, O. A. Yakimenko, J. F. Whidborne, and A. K. Cooke, "A prototype of an autonomous controller for a quadrotor UAV," in ECC '07, 2007.

[8] H. Voos, "Nonlinear control of a quadrotor micro-uav using feedbacklinearization," in ICM '09, 2009, pp. 1-6.

[9] T. Lee, M. Leok, and N. H. McClamroch, "Geometric Tracking Control of a Quadrotor UAV on $\mathrm{SE}(3)$," in $C D C$ ' 10 , Atlanta, GA, USA, December 2010.

[10] T. Madani and A. Benallegue, "Backstepping control for a quadrotor helicopter," in IROS '06, 2006, pp. 3255-3260.

[11] S. Bouabdallah and R. Siegwart, "Backstepping and Sliding-mode Techniques Applied to an Indoor Micro Quadrotor," in ICRA '05, 2005, pp. $2247-2252$.

[12] G. V. Raffo, M. G. Ortega, and F. R. Rubio, "An Integral Predictive/Nonlinear Control Structure for a Quadrotor Helicopter," Automatica, vol. 46, no. 1, pp. 29 - 39, 2010.

[13] K. Alexis, G. Nikolakopoulos, and A. Tzes, "Experimental model predictive attitude tracking control of a quadrotor helicopter subject to wind-gusts," in $M E D$ ' 10,2010 , pp. 1461-1466.

[14] Z. T. Dydek, A. M. Annaswamy, and E. Lavretsky, "Adaptive Control of Quadrotor UAVs: A Design Trade Study With Flight Evaluations," Control Systems Technology, IEEE Transactions on, vol. PP, no. 99, p. $1,2012$.

[15] D. Mellinger, Q. Lindsey, M. Shomin, and V. Kumar, "Design, modeling, estimation and control for aerial grasping and manipulation," in IROS '11, 2011, pp. 2668-2673.

[16] L. Besnard, Y. Shtessel, and B. Landrum, "Control of a quadrotor vehicle using sliding mode disturbance observer,' in ACC '07, 2007, pp. 5230-5235.

[17] T. Madani and A. Benallegue, "Sliding Mode Observer and Backstepping Control for a Quadrotor Unmanned Aerial Vehicles," in ACC'07, 2007, pp. 5887-5892.

[18] Y. Al-Younes, M. Jarrah, and S. Sukkarieh, "Adaptive integral backstepping controller for an autonomous rotorcraft," in ISMA '09, 2009, pp. $1-7$.

[19] Z. Fang and W. Gao, "Adaptive integral backstepping control of a micro-quadrotor," in ICICIP '11, vol. 2, 2011, pp. 910-915.

[20] J. Escareo, S. Salazar, H. Romero, and R. Lozano, "Trajectory Control of a Quadrotor Subject to 2D Wind Disturbances," Journal of Intelligent \& Robotic Systems, pp. 1-13, 10.1007/s10846-012-9734-1.

[21] F. Leonard, A. Martini, and G. Abba, "Robust nonlinear controls of model-scale helicopters under lateral and vertical wind gusts," Control Systems Technology, IEEE Transactions on, vol. 20, no. 1, pp. 154163, Jan. 2012.

[22] X. Gong, Y. Tian, Y. Bai, and C. Zhao, "Trajectory tacking control of a quad-rotor based on active disturbance rejection control," in ICAL '12, 2012, pp. 254-259.

[23] C. Hancer, K. Oner, E. Sirimoglu, E. Cetinsoy, and M. Unel, "Robust position control of a tilt-wing quadrotor," in CDC '10, 2010, pp. 49084913.

[24] J.-Y. Park, B.-H. Cho, and J.-K. Lee, "Trajectory-tracking control of underwater inspection robot for nuclear reactor internals using time delay control," Nuclear Engineering and Design, vol. 239, pp. 25432550,2009

[25] K. Kondak, M. Bernard, N. Meyer, and G. Hommel, "Autonomously Flying VTOL-Robots: Modeling and Control," in ICRA '07, 2007, pp. 736-741.

[26] K. Lee, J. Back, and I. Choy, "Disturbance Observer Based Trajectory Tracking Controller for Quadrotor," in ICCAS '12, 2012, pp. 157-161.

[27] S.-H. Jeong and S. Jung, "Experimental studies of a disturbance observer for attitude control of a quad-rotor system,' in ICCAS '12, 2012, pp. 579-583.

[28] S. Jeong, S. Jung, and M. Tomizuka, "Attitude control of a quad-rotor system using an acceleration-based disturbance observer: An empirical approach," in AIM '12, 2012, pp. 916-921.

[29] S.-U. Lee and P. H. Chang, "The Development of Anti-Windup Scheme for Time Delay Control With Switching Action Using Integral Sliding Surface," Journal of Dynamic Systems, Measurement, and Control, vol. 125, p. 630, 2003. 